\section{New Theory}

ISSN: 2149-1402
35 (2021) 62-71

Journal of New Theory

https://dergipark.org.tr/en/pub/jnt

Open Access

\title{
Semi-Invariant Riemannian Submersions with Semi-Symmetric Non-Metric Connection
}

\author{
Ramazan Sarı ${ }^{1}$
}

\author{
Article History \\ Received: 28 Mar 2021 \\ Accepted: 07 Jun 2021 \\ Published: 30 Jun 2021 \\ Research Article
}

\begin{abstract}
In this paper, we investigate semi-invariant Riemannian submersion from a Kaehler manifold with semi-symmetric non-metric connection to a Riemannian manifold. We study the geometry of foliations with semi-symmetric non-metric connection. Later, we introduce base manifold to be a local product manifold with semi-symmetric non-metric connection.
\end{abstract}

Keywords - Riemannian submersions, semi-invariant submersions, semi-symmetric non-metric connection, Kaehler manifold

Mathematics Subject Classification (2020) - 53C15, 53C40

\section{Introduction}

A conventional way to compare two manifolds is by defining smooth maps from one manifold to another. One such map is submersion, whose rank equals to the dimension of the target manifold. Riemannian submersion between Riemannian submanifolds were first introduced by O' Neill and Gray $[1,2]$. Later many authors studied different geometric properties of the Riemannian submersions [3], semi-slant submersions [4-6], hemi-slant submersions [7-9], semi-invariant submersions [10-12], antiinvariant submersions [13-15].

On the other hand, Friedmann et al. defined the concept of the semi-symmetric non-metric connection in a differential manifold [16]. Hayden studied metric connection with torsion a Riemannian manifold [17]. Later, Yano investigated a Riemannian manifold with new connection, which is called a semi-symmetric metric connection [18]. Afterwards, Agashe et al. studied semi-symmetric non-metric connection (SSNMC) on a Riemannian manifold [19]. Many author have studied semi-symmetric connection [20-26].

Let $M$ be differentiable manifold with linear connection $\nabla$. Therefore, for all $K, L \in \Gamma(T N)$, we get

$$
T(K, L)=\nabla_{K} L-\nabla_{L} K-[K, L]
$$

where $T$ is torsion tensor of $\nabla$. If the torsion tensor $T=0$, then the connection $\nabla$ is said to be symmetric, otherwise it is called non-symmetric. Moreover, for all $K, L \in \Gamma(T N)$, the connection $\nabla$ is said to be semi-symmetric if

$$
T(K, L)=\eta(L) K-\eta(K) L
$$

where $\eta$ is a 1 -form on $N$. However, $\nabla$ is called metric connection if $\nabla g=0$ with Riemannian metric $g$, otherwise it is said to be non-metric.

\footnotetext{
${ }^{1}$ ramazan.sari@amasya.edu.tr (Corresponding Author)

${ }^{1}$ Gümüşhacıköy Hasan Duman Vocational School, Amasya University, Amasya, Turkey
} 
In [27], Akyol and Beyendi studied the idea of Riemannian submersion with SSNMC. They investigated O'Neill's tensor fields, obtain derivatives of those tensor fields and compare curvatures of the total manifold, the base manifold and the fibres by computing curvatures.

The main purpose of this paper is to investigate geometry of semi-invariant Riemannian submersion from a Kaehler manifold with SSNMC to a Riemannian manifold.

\section{Preliminaries}

Definition 2.1. Let $F:\left(N^{n}, g_{N}\right) \rightarrow\left(B^{b}, g_{B}\right)$ be a submersion between two Riemannian manifolds. Then, $F$ said to be Riemannian submersion if

i. $F$ has maximal rank.

ii. The differential $F_{*}$ preserves the lenghts of horizontal vectors.

On the other hand, $F^{-1}(k)$ is an $(n-b)$ dimensional submanifold of $N$, for each $k \in N$ The submanifolds $F^{-1}(k)$ are called fibers. Moreover, vector fields tangent to fibers are called vertical and vector fields orthogonal to fibers are horizantal. A vector field $X$ on $N$ is called basic if $X$ is horizontal and $F_{*} X_{q}=X_{\pi_{*}(q)}$ for all $q \in N$. We determine that $\mathcal{V}$ and $\mathcal{H}$ define projections $k e r F_{*}$ and $\left(k e r F_{*}\right)^{\perp}$, respectively.

On the other hand, a Riemannian submersion $F: N \rightarrow B$ determines tensor fields $T$ and $A$ on $N$ such that,

$$
\begin{aligned}
& T(E, F)=T_{E} F=\mathcal{H} \nabla_{\mathcal{V} E}^{M} \mathcal{V} F+\mathcal{V} \nabla_{\mathcal{V} E}^{M} \mathcal{H} F, \\
& A(E, F)=A_{E} F=\mathcal{V} \nabla_{\mathcal{H} E}^{M} \mathcal{H} F+\mathcal{H} \nabla_{\mathcal{H} E}^{M} \mathcal{V} F
\end{aligned}
$$

for any $E, F \in \Gamma(T M)$ (see [1]). By virtue of (1) and (2), one can obtain

$$
\begin{gathered}
\nabla_{V}^{M} W=T_{V} W+\hat{\nabla}_{V} W \\
\nabla_{V}^{M} X=T_{V} X+\mathcal{H}\left(\nabla_{V}^{M} X\right) \\
\nabla_{X}^{M} V=\mathcal{V}\left(\nabla_{X}^{M} V\right)+A_{X} V \\
\nabla_{X}^{M} Y=A_{X} Y+\mathcal{H}\left(\nabla_{X}^{M} Y\right)
\end{gathered}
$$

for all $V, W \in \Gamma\left(k e r F_{*}\right)$ and $X, Y \in \Gamma\left(\left(\operatorname{ker} F_{*}\right)^{\perp}\right)$. Further, if $X$ is basic, then

$$
\mathcal{H}\left(\nabla_{V}^{M} X\right)=A_{X} V
$$

On the other hand, let $N, B$ be two Rieamannian manifold and $F: N \rightarrow B$ is a smooth map. Therefore, the second fundamental form of $F$ is expressed by

$$
\left(\nabla F_{*}\right)(K, L)=\nabla_{K}^{B} F_{*} L-F_{*}\left(\nabla_{K}^{N} L\right)
$$

for $K, L \in \Gamma(T N)$. Moreover, $\pi$ is said to be a totally geodesic map if $\left(\nabla F_{*}\right)(K, L)=0$ for $K, L \in$ $\Gamma(T N)[28]$.

Now, we recall the definition of Kaehler manifold. Let $N$ be a Hermitian manifold with respect Hermitian structure $(J, g)$ such that

$$
J^{2}=-I
$$

and

$$
g(E, F)=g(J E, J F)
$$

for all $E, F \in \Gamma(T N)$, where $g(J E, F)=-g(E, J F)$.

A Hermitian manifold is called Kaehler manifold if

$$
\nabla J=0
$$


On the other hand, we define a linear connection $\widetilde{\nabla}$ on Kaehler manifold $N$ such that

$$
\widetilde{\nabla}_{E} F=\nabla_{E} F+\eta(F) E
$$

where $E, F \in \Gamma(T N), \nabla$ is a Levi-Civita connection on $\mathrm{N}$ and $\eta$ is a 1-form with the vector field $P$ on $N$ by

$$
\eta(E)=g(E, P)
$$

By virute of (12), we arrive that

$$
\widetilde{T}(E, F)=\eta(F) E-\eta(E) F
$$

and

$$
\left(\widetilde{\nabla}_{E} g\right)(F, K)=-\eta(F) g(E, K)-\eta(K) g(E, F)
$$

where $\widetilde{T}$ is torsion tensor of $\widetilde{\nabla}$. Then, $\widetilde{\nabla}$ defined a semi-symmetric non metric conection with (12).

Let $\mathrm{N}$ be a Kaehler manifold. We using (12), for all $K, L \in \Gamma(T N)$, we get,

$$
\begin{aligned}
\left(\nabla_{K} J\right) L & =\nabla_{K} J L-J \nabla_{K} L \\
& =\widetilde{\nabla}_{K} J L-\eta(L) K-J \widetilde{\nabla}_{K} L+\eta(L) J K
\end{aligned}
$$

Then, using (11) we obtain,

$$
\left(\widetilde{\nabla}_{K} J\right) L=\eta(L) J K-\eta(L) K
$$

Now, we call O'Neill's tensor fields for SSNMC [27]. For all $K, L \in \Gamma(T N)$, we have,

$$
\widetilde{T}_{K} L=T_{K} L+\eta(h L) v K
$$

and

$$
\widetilde{A}_{K} L=A_{K} L+\eta(v L) h K
$$

Then, using last two equations, we obtain

$$
\begin{gathered}
\widetilde{\nabla}_{K} L=T_{K} L+v \widetilde{\nabla}_{K} L \\
\widetilde{\nabla}_{K} X=T_{K} X+h \widetilde{\nabla}_{K} X+\eta(X) K \\
\widetilde{\nabla}_{X} K=A_{X} K+v \widetilde{\nabla}_{X} K+\eta(K) X \\
\widetilde{\nabla}_{X} Y=A_{X} Y+h \widetilde{\nabla}_{X} Y
\end{gathered}
$$

where for all $K, L \in \Gamma\left(k e r F_{*}\right), X, Y \in \Gamma\left(\left(\operatorname{ker} F_{*}\right)^{\perp}\right)$.

\section{Semi-Invariant Riemannian Submersion}

Definition 3.1. Let $N$ and $B$ be a Kaehler manifold and Riemannian manifold, respectively. Let us assume that $F: N \rightarrow B$ be a Riemannian submersion. Therefore, $F$ is called semi-invariant Riemannian submersion if there is a distribution $D_{1} \subseteq k e r F_{*}$ such that

$$
\operatorname{ker} F_{*}=D_{1} \oplus D_{2}
$$

and

$$
J D_{1}=D_{1}, \quad J D_{2} \subseteq\left(\operatorname{ker} F_{*}\right)^{\perp}
$$

where $D_{2}$ is orthogonal complementary to $D_{1}$ in $\operatorname{ker} F_{*}$ ( [12]). 
Example 3.2. Let $F$ be a submersion. We denote that

$$
F: \begin{gathered}
\mathbb{R}^{6} \\
\left(x_{1}, x_{2}, x_{3}, x_{4}, x_{5}, x_{6}\right)
\end{gathered} \longrightarrow \begin{gathered}
\mathbb{R}^{3} \\
\left(\frac{x_{1}+x_{2}}{\sqrt{2}}, \frac{x_{3}+x_{6}}{\sqrt{2}}, \frac{x_{4}+x_{5}}{\sqrt{2}}\right)
\end{gathered}
$$

Then, it follows that

$$
k e r F_{*}=\operatorname{span}\left\{V_{1}=\frac{\partial}{\partial x_{1}}-\frac{\partial}{\partial x_{2}}, V_{2}=\frac{\partial}{\partial x_{3}}-\frac{\partial}{\partial x_{6}}, V_{3}=-\frac{\partial}{\partial x_{4}}-\frac{\partial}{\partial x_{5}}\right\}
$$

and

$$
\left(k e r F_{*}\right)^{\perp}=\operatorname{span}\left\{H_{1}=\frac{\partial}{\partial x_{1}}+\frac{\partial}{\partial x_{2}}, H_{2}=\frac{\partial}{\partial x_{3}}+\frac{\partial}{\partial x_{6}}, H_{3}=\frac{\partial}{\partial x_{4}}+\frac{\partial}{\partial x_{5}}\right.
$$

Hence we have $J V_{1}=-V_{1}, J V_{2}=H_{3}$ and $J V_{3}=-H_{2}$. Thus it follows that $D_{1}=\operatorname{span}\left\{V_{1}\right\}$ and $D_{2}=\operatorname{span}\left\{H_{2}, H_{3}\right\}$. On the other hand, we arrive that,

$$
g_{\mathbb{R}^{3}}\left(F_{*} H_{2}, F_{*} H_{2}\right)=g_{\mathbb{R}^{6}}\left(H_{2}, H_{2}\right), \quad g_{\mathbb{R}^{3}}\left(F_{*} H_{3}, F_{*} H_{3}\right)=g_{\mathbb{R}^{6}}\left(H_{3}, H_{3}\right)
$$

where $g_{\mathbb{R}^{3}}$ and $g_{\mathbb{R}^{6}}$ determine metrics of $\mathbb{R}^{3}$ and $\mathbb{R}^{6}$, respectively. Then, $F$ is semi-invariant Riemannian submersion.

Let $F:(N, J, g) \rightarrow(B, g)$ be a semi-invariant Riemannian submerion such that $N$ and $B$ are Kaehler manifold and Riemannian manifold respectively. For all $K \in \Gamma(T N)$, we write

$$
E=\mathcal{V} E+\mathcal{H} E
$$

where $\mathcal{V} E \in \Gamma\left(k e r F_{*}\right)$ and $\mathcal{H} E \in \Gamma\left(\left(\operatorname{ker} F_{*}\right)^{\perp}\right)$. Then, for all $K \in \Gamma\left(k e r F_{*}\right)$, we write

$$
J K=\phi K+\omega K
$$

where $\phi K \in \Gamma\left(D_{1}\right)$ and $\omega K \in \Gamma\left(J D_{2}\right)$.

Since $F$ is a semi-invariant Riemannian submersion, we can determine

$$
\left(\operatorname{ker} F_{*}\right)^{\perp}=J D_{2} \oplus \mu
$$

where $J D_{2}$ and $\mu$ are complementary to each other. Similarly, $x \in \Gamma\left(\left(\operatorname{ker} F_{*}\right)^{\perp}\right)$, we get

$$
J X=B X+C X
$$

where $B X \in \Gamma\left(D_{2}\right)$ and $C X \in \Gamma(\mu)$.

\section{Geometry of Distributions}

We note that, for brevity we use a abbreviation " $F$ is a semi-invariant Riemannian submersion with SSNMC" for $F:(N, J, g) \rightarrow(B, g)$ be a semi-invariant Riemannian submersion from Kaehler manifold with SSNMC $M$ and Riemannian manifold $N$.

Theorem 4.1. Let $F$ be a semi-invariant Riemannian submersion with SSNMC. Therefore, the distribution $D_{1}$ is integrable if and only if we have

$$
\begin{aligned}
g_{B}\left(F_{*}\left(T_{V} B Z+h \widetilde{\nabla}_{V} C Z\right), F_{*}(\omega U)\right)-g_{B}\left(F_{*}\left(T_{U} B Z+h \widetilde{\nabla}_{U} C Z\right), F_{*}(\omega V)\right)= & g_{N}\left(v \widetilde{\nabla}_{U} B Z+T_{U} C Z, \phi V\right) \\
& -g_{N}\left(v \widetilde{\nabla}_{V} B Z+T_{V} C Z, \phi U\right) \\
& +2 g_{N}(\phi U, V) \eta(Z)
\end{aligned}
$$

for all $U, V \in \Gamma\left(D_{1}\right)$ and $Z \in \Gamma\left(D_{2}\right)$. 
Proof. Firstly, we using (10) and (13). For all $U, V \in \Gamma\left(D_{1}\right)$ and $Z \in \Gamma\left(D_{2}\right)$, we arrive that

$$
g_{N}\left(\widetilde{\nabla}_{U} V, Z\right)=g_{N}\left(\widetilde{\nabla}_{U} J V, J Z\right)
$$

By virtue of (13) and (20), we get,

$$
g_{N}([U, V], Z)=g_{N}\left(\widetilde{\nabla}_{U} J V, J Z\right)-g_{N}\left(\widetilde{\nabla}_{V} J U, J Z\right)
$$

After some calculations, we conclude,

$$
g_{N}([U, V], Z)=-g_{N}\left(J V, \widetilde{\nabla}_{U} J Z\right)+g_{N}\left(J U, \widetilde{\nabla}_{V} J Z\right)
$$

We know that $F$ is a semi-invariant Riemannian submersion, by virtue of (19), (14), (15) and (18), we conclude that,

$$
\begin{aligned}
g_{N}([U, V], Z)= & -g_{N}\left(\phi V, v \widetilde{\nabla}_{U} B Z+T_{U} C Z\right)-g_{N}\left(w V, T_{U} B Z+h \widetilde{\nabla}_{U} C Z\right)-\eta(C Z) g_{N}(J V, U) \\
& +g_{N}\left(\phi U, v \widetilde{\nabla}_{V} B Z+T_{V} C Z\right)+g_{N}\left(w U, T_{V} B Z+h \widetilde{\nabla}_{V} C Z\right)+\eta(C Z) g_{N}(J U, V)
\end{aligned}
$$

which gives proof.

Theorem 4.2. Let $F$ be a semi-invariant Riemannian submersion with SSNMC . Therefore, the distribution $D_{2}$ is integrable if and only if we have

$$
g_{B}\left(F_{*}\left(T_{Z} \phi U+h \widetilde{\nabla}_{Z} w U\right), F_{*}(J W)\right)=g_{B}\left(F_{*}\left(T_{W} \phi U+h \widetilde{\nabla}_{W} w U\right), F_{*}(J Z)\right)
$$

for all $U \in \Gamma\left(D_{1}\right)$ and $Z, W \in \Gamma\left(D_{2}\right)$.

Proof. By virtue of (12), (10) and (13), we get

$$
g_{N}([Z, W], U)=g_{N}\left(\widetilde{\nabla}_{Z} J W, J U\right)-g_{N}\left(\widetilde{\nabla}_{W} J Z, J U\right)
$$

for all $U \in \Gamma\left(D_{1}\right)$ and $Z, W \in \Gamma\left(D_{2}\right)$. Therefore, we conclude

$$
g_{N}([Z, W], U)=-g_{N}\left(J W, \widetilde{\nabla}_{Z} J U\right)+g_{N}\left(J Z, \widetilde{\nabla}_{W} J U\right)
$$

Then, using (18), (14) and (15), we arrive,

$$
g_{N}([Z, W], U)=-g_{N}\left(J W, T_{Z} \phi U+h \widetilde{\nabla}_{Z} w U\right)+g_{N}\left(J Z, T_{W} \phi U+h \widetilde{\nabla}_{W} w U\right)
$$

which proves assertion.

Theorem 4.3. Let $F$ be a semi-invariant Riemannian submersion with SSNMC. Therefore, the distribution $D_{1}$ defines a totally geodesic foliation on $N$ if and only if we have

$$
F_{*}\left(\widetilde{\nabla}_{U} J V\right) \in \Gamma(\mu)
$$

and

$$
\begin{aligned}
g_{B}\left(F_{*}\left(T_{U} \phi V\right), F_{*}(C X)\right)+g_{B}\left(F_{*}\left(h \widetilde{\nabla}_{U} w V\right), F_{*}(C X)\right)= & g_{N}\left(v \widetilde{\nabla}_{U} \phi V+T_{U} w V, B X\right) \\
& +g_{N}(U, B X) \eta(w V)
\end{aligned}
$$

for all $U, V \in \Gamma\left(D_{1}\right), Z \in \Gamma\left(D_{2}\right)$ and $X \in \Gamma\left(\operatorname{ker} F_{*}^{\perp}\right)$.

Proof. We know that, $D_{1}$ defines a totally geodesic foliation on $M$ if and only if $g_{N}\left(\widetilde{\nabla}_{U} V, Z\right)=0$ and $g_{N}\left(\widetilde{\nabla}_{U} V, X\right)=0$, for all $U, V \in \Gamma\left(D_{1}\right), Z \in \Gamma\left(D_{2}\right)$ and $X \in \Gamma\left(\operatorname{ker} F_{*}^{\perp}\right)$.

Then, using (13) and (10), we get,

$$
g_{N}\left(\widetilde{\nabla}_{U} V, Z\right)=g_{N}\left(\widetilde{\nabla}_{U} J V, J Z\right)
$$


Since $E=\mathcal{V} E+\mathcal{H} E$, for all $E \in \Gamma(T M)$, we have

$$
g_{N}\left(\widetilde{\nabla}_{U} V, Z\right)=g_{N}\left(\mathcal{H} \widetilde{\nabla}_{U} J V, J Z\right)
$$

Therefore, $F$ is a semi-invariant Riemannian submersion and character of $F$, we arrive that,

$$
g_{N}\left(\widetilde{\nabla}_{U} V, Z\right)=g_{B}\left(F_{*}\left(\mathcal{H} \widetilde{\nabla}_{U} J V\right), F_{*}(J Z)\right)
$$

Moreover, using (13) and (10), we get

$$
g_{N}\left(\widetilde{\nabla}_{U} V, X\right)=g_{N}\left(\widetilde{\nabla}_{U} J V, J X\right)
$$

By virtue of (14) and (15), we get

$$
g_{N}\left(\widetilde{\nabla}_{U} V, X\right)=g_{N}\left(T_{U} \phi V+h \widetilde{\nabla}_{U} w V, C X\right)+g_{N}\left(v \widetilde{\nabla}_{U} \phi V, B X\right)+\eta(w V) g_{N}(U, B X)
$$

or

$$
g_{N}\left(\widetilde{\nabla}_{U} V, X\right)=g_{B}\left(F_{*}\left(T_{U} \phi V+h \widetilde{\nabla}_{U} w V\right), F_{*}(C X)\right)+g_{M}\left(v \widetilde{\nabla}_{U} \phi V, B X\right)+\eta(w V) g_{N}(U, B X)
$$

which gives our assertion.

Theorem 4.4. Let $F$ be a semi-invariant Riemannian submersion with SSNMC. Therefore, the distribution $D_{2}$ defines a totally geodesic foliation on $N$ if and only if we have

$$
g_{B}\left(F_{*}\left(T_{Z} B X\right), F_{*}(C X)\right)+g_{B}\left(F_{*}\left(h \widetilde{\nabla}_{Z} C X\right), F_{*}(C X)\right)=-g_{N}\left(v \widetilde{\nabla}_{Z} B X+T_{Z} C X, B X\right)-\eta(C X) g_{N}(Z, B X)
$$

and

$$
\begin{aligned}
g_{B}\left(F_{*}\left(T_{Z} \phi U\right), F_{*}(C W)\right)+g_{B}\left(F_{*}\left(h \widetilde{\nabla}_{Z} w U\right), F_{*}(C W)\right)= & g_{N}\left(v \widetilde{\nabla}_{Z} \phi U+T_{Z} w U, B W\right) \\
& +g_{N}(Z, B W) \eta(w U)-g_{N}(Z, \phi U) \eta(w W)
\end{aligned}
$$

for all $Z, W \in \Gamma\left(D_{2}\right), U \in \Gamma\left(D_{1}\right)$ and $X \in \Gamma\left(\operatorname{ker} F_{*}^{\perp}\right)$.

Proof. For all $Z, W \in \Gamma\left(D_{2}\right), X \in \Gamma\left(\operatorname{ker} F_{*}^{\perp}\right)$, using (10), and (13), we conclude,

$$
g_{N}\left(\widetilde{\nabla}_{Z} W, X\right)=g_{N}\left(\widetilde{\nabla}_{Z} J W, J X\right)
$$

Then, from (19), (14) and (15), we have,

$$
g_{N}\left(\widetilde{\nabla}_{Z} W, X\right)=g_{N}\left(T_{Z} B X+v \widetilde{\nabla}_{Z} B X+T_{Z} C X+h \widetilde{\nabla}_{Z} C X+\eta(C X) Z, B X+C X\right)
$$

We know that $F$ is semi-invariant Riemannian submersion, we conclude,

$g_{N}\left(\widetilde{\nabla}_{Z} W, X\right)=g_{B}\left(F_{*}\left(T_{Z} B X+h \widetilde{\nabla}_{Z} C X\right), F_{*}(C X)\right)+g_{N}\left(v \widetilde{\nabla}_{Z} B X+T_{Z} C X, B X\right)+\eta(C X) g_{N}(Z, B X)$

Moreover, for all $Z, W \in \Gamma\left(D_{2}\right), U \in \Gamma\left(D_{1}\right)$, using (10), and (13),

$$
g_{N}\left(\widetilde{\nabla}_{Z} W, U\right)=-g_{N}\left(J W, \widetilde{\nabla}_{Z} J U\right)
$$

By virtue of (19), (14) and (15), imply that

$$
g_{N}\left(\widetilde{\nabla}_{Z} W, U\right)=-g_{N}\left(B W+C W, T_{Z} \phi U+v \widetilde{\nabla}_{Z} \phi U+T_{Z} w U+h \widetilde{\nabla}_{Z} w U+\eta(w U) Z\right)-\eta(J W) g_{N}(Z, J U)
$$

Since $F$ is semi-invariant Riemannian submersion, we arrive,

$$
\begin{aligned}
g_{N}\left(\widetilde{\nabla}_{Z} W, U\right)= & -g_{B}\left(F_{*}\left(T_{Z} \phi U+h \widetilde{\nabla}_{Z} w U\right), F_{*}(C W)\right)-g_{N}\left(B W, v \widetilde{\nabla}_{Z} \phi U+T_{Z} w U\right) \\
& -\eta(w U) g_{N}(B W, Z)-\eta(w W) g_{N}(Z, \phi U)
\end{aligned}
$$

which give proof. 
Corollary 4.5. Let $F$ be a semi-invariant Riemannian submersion with SSNMC. Therefore, the fibers of $F$ are the locally product Riemannian manifold of leaves of $D_{1}$ and $D_{2}$ if and only if

$$
\begin{array}{cc}
F_{*}\left(\widetilde{\nabla}_{U} J V\right) \in \Gamma(\mu), & \\
g_{B}\left(F_{*}\left(T_{U} \phi V\right), F_{*}(C X)\right)+g_{B}\left(F_{*}\left(h \widetilde{\nabla}_{U} w V\right), F_{*}(C X)\right)= & g_{N}\left(v \widetilde{\nabla}_{U} \phi V+T_{U} w V, B X\right) \\
& +g_{N}(U, B X) \eta(w V)
\end{array}
$$

and

$$
\begin{aligned}
g_{B}\left(F_{*}\left(T_{Z} B X\right), F_{*}(C X)\right)+g_{B}\left(F_{*}\left(h \widetilde{\nabla}_{Z} C X\right), F_{*}(C X)\right)=- & g_{N}\left(v \widetilde{\nabla}_{Z} B X+T_{Z} C X, B X\right)-\eta(C X) g_{N}(Z, B X) \\
g_{B}\left(F_{*}\left(T_{Z} \phi U\right), F_{*}(C W)\right)+g_{B}\left(F_{*}\left(h \widetilde{\nabla}_{Z} w U\right), F_{*}(C W)\right)= & g_{N}\left(v \widetilde{\nabla}_{Z} \phi U+T_{Z} w U, B W\right) \\
& +g_{N}(Z, B W) \eta(w U)-g_{M}(Z, \phi U) \eta(w W)
\end{aligned}
$$

for all $U, V \in \Gamma\left(D_{1}\right), Z, W \in \Gamma\left(D_{2}\right)$ and $X \in \Gamma\left(\operatorname{ker} F_{*}^{\perp}\right)$.

Theorem 4.6. Let $F$ be a semi-invariant Riemannian submersion with SSNMC. Therefore, the distribution $\operatorname{ker} F_{*}^{\perp}$ is integrable if and only if we have

$$
A_{Y} C X-A_{X} C Y+v \widetilde{\nabla}_{Y} B X-v \widetilde{\nabla}_{X} B Y \notin \Gamma\left(D_{1}\right)
$$

and

$$
\begin{aligned}
g_{B}\left(F_{*}\left(A_{Y} B X\right), F_{*}(w Z)\right)+g_{B}\left(F_{*}\left(h \widetilde{\nabla}_{Y} C X, F_{*}(w Z)\right)=\right. & -g_{N}\left(v \widetilde{\nabla}_{Y} B X+A_{Y} C X, \phi Z\right) \\
& +\eta(X) g_{N}(Y-w Y, w Z)-\eta(Y) g_{N}(X-w X, w Z)
\end{aligned}
$$

for all $X \in \Gamma\left(\operatorname{ker} F_{*}^{\perp}\right), Z \in \Gamma\left(D_{2}\right)$ and $U \in \Gamma\left(D_{1}\right)$.

Proof. We using (12), (10) and (13), for all $X, Y \in \Gamma\left(\operatorname{ker} F_{*}^{\perp}\right), U \in \Gamma\left(D_{1}\right)$, we have

$$
g_{N}([X, Y], U)=g_{N}\left(\widetilde{\nabla}_{X} J Y, J U\right)-g_{N}\left(\widetilde{\nabla}_{Y} J X, J U\right)
$$

Then, using (19), (16) and (17), we arrive,

$$
g_{N}([X, Y], U)=-g_{N}\left(-v \widetilde{\nabla}_{X} B Y-A_{X} C Y+v \widetilde{\nabla}_{Y} B X+A_{Y} C X, U\right)
$$

Moreover, for $Z \in \Gamma\left(D_{2}\right)$, by (12), (10) and (13), we get

$$
\begin{aligned}
g_{N}([X, Y], Z)= & g_{N}\left(\widetilde{\nabla}_{X} J Y, J Z\right)-\eta(Y) g_{N}(X-J X, J Z) \\
& -g_{N}\left(\widetilde{\nabla}_{Y} J X, J Z\right)+\eta(X) g_{N}(Y-J Y, J Z)
\end{aligned}
$$

Therefore, by virtue of (18), (15) and (16), we conclude that

$$
\begin{aligned}
g_{N}([X, Y], Z)= & -g_{N}\left(A_{Y} B X+h \widetilde{\nabla}_{Y} C X, w Z\right)-g_{N}\left(v \widetilde{\nabla}_{Y} B X+A_{Y} C X, \phi Z\right) \\
& -\eta(Y) g_{N}(X-w X, w Z)+\eta(X) g_{N}(Y-w Y, w Z)
\end{aligned}
$$

On the other hand, $F$ is semi-invariant Riemannian submersion, therefore we get,

$$
\begin{aligned}
g_{N}([X, Y], Z)= & -g_{B}\left(F_{*}\left(A_{Y} B X+h \widetilde{\nabla}_{Y} C X\right), F_{*}(w Z)\right)-g_{N}\left(v \widetilde{\nabla}_{Y} B X+A_{Y} C X, \phi Z\right) \\
& -\eta(Y) g_{N}(X-w X, w Z)+\eta(X) g_{N}(Y-w Y, w Z)
\end{aligned}
$$

which conclude proof. 
Theorem 4.7. Let $F$ be a semi-invariant Riemannian submersion with SSNMC . Therefore, the distribution ker $F_{*}^{\perp}$ defines a totally geodesic foliation on $N$ if and only if we have

$$
v \widetilde{\nabla}_{X} B Y+A_{X} C Y \in \Gamma\left(D_{2}\right)
$$

and

$$
g_{B}\left(F_{*}\left(h \widetilde{\nabla}_{X} C Y\right), F_{*}(w Z)\right)=-g_{N}\left(A_{X} B Y, J Z\right)
$$

for all $X, Y \in \Gamma\left(\operatorname{ker} F_{*}^{\perp}\right), U \in \Gamma\left(D_{1}\right)$ and $Z \in \Gamma\left(D_{2}\right)$.

Proof. We using (10), (13) and (19), for all $X, Y \in \Gamma\left(\operatorname{ker} F_{*}^{\perp}\right), U \in \Gamma\left(D_{1}\right)$, we have

$$
g_{N}\left(\widetilde{\nabla}_{X} Y, U\right)=g_{N}\left(\widetilde{\nabla}_{X} B Y, J U\right)+g_{M}\left(\widetilde{\nabla}_{X} C Y, J U\right)
$$

Therefore, using (15), (16) and (10), we arrive

$$
g_{N}\left(\widetilde{\nabla}_{X} Y, U\right)=-g_{N}\left(J\left(v \widetilde{\nabla}_{X} B Y+A_{X} C Y\right), U\right)
$$

Moreover, for $Z \in \Gamma\left(D_{2}\right)$, using (10), (19),(16) and (17), we conclude,

$$
g_{N}\left(\widetilde{\nabla}_{X} Y, Z\right)=g_{N}\left(A_{X} B Y+v \widetilde{\nabla}_{X} B Y+\eta(B Y) X+A_{X} C Y+h \widetilde{\nabla}_{X} C Y, J Z\right)
$$

Also, character of $F$, we obtain

$$
g_{N}\left(\widetilde{\nabla}_{X} Y, Z\right)=g_{B}\left(F_{*}\left(h \widetilde{\nabla}_{X} C Y\right), F_{*}(w Z)\right)+g_{N}\left(A_{X} B Y, J Z\right)
$$

which completes proof.

Theorem 4.8. Let $F$ be a semi-invariant Riemannian submersion with SSNMC. Therefore, the distribution $\operatorname{ker} F_{*}$ defines a totally geodesic foliation on $N$ if and only if we have

$$
v \widetilde{\nabla}_{K} L+T_{K} w L+\eta(w L) E \in \Gamma\left(D_{1}\right)
$$

and

$$
\begin{aligned}
g_{B}\left(F_{*}\left(T_{K} \phi L\right), F_{*}(C X)\right)+g_{B}\left(F_{*}\left(h \widetilde{\nabla}_{K} w L\right), F_{*}(C X)\right)= & -g_{N}\left(v \widetilde{\nabla}_{K} \phi L, B X\right)-g_{N}\left(T_{K} w L, B X\right) \\
& -\eta(\phi L) g_{N}(K, B X)
\end{aligned}
$$

for all $K, L \in \Gamma\left(\operatorname{ker} F_{*}\right)$ and $X \in \Gamma(\mu)$.

Proof. We know that ker $F_{*}$ denote a totally geodesic foliation on $N$ if and only if $g_{N}\left(\widetilde{\nabla}_{K} L, X\right)=0$ and $g_{N}\left(\widetilde{\nabla}_{K} L, J Z\right)=0$ for all $Z \in \Gamma\left(D_{2}\right), K, L \in \Gamma\left(\operatorname{ker} F_{*}\right)$ and $X \in \Gamma(\mu)$.

Then, by (10), (13) and (18), we get

$$
g_{N}\left(\widetilde{\nabla}_{K} L, X\right)=g_{N}\left(\widetilde{\nabla}_{K} \phi L, J X\right)+g_{N}\left(\widetilde{\nabla}_{K} w L, J X\right)
$$

Therefore, using (14), (15) and (19), we have

$$
g_{N}\left(\widetilde{\nabla}_{K} L, X\right)=g_{N}\left(v \widetilde{\nabla}_{K} \phi L+T_{K} w L+\eta(\phi L) K, B X\right)+g_{N}\left(T_{K} \phi L+h \widetilde{\nabla}_{K} w L, C X\right)
$$

We know that $F$ is semi-invariant Riemannian submersion, we arrive

$$
g_{N}\left(\widetilde{\nabla}_{K} L, X\right)=g_{N}\left(v \widetilde{\nabla}_{K} \phi L+T_{K} w L+\eta(\phi L) K, B X\right)+g_{B}\left(F_{*}\left(T_{K} \phi L+h \widetilde{\nabla}_{K} w L\right), F_{*}(C X)\right)
$$

Moreover, from (10), (13) ,(18), (14) and (15), we obtain,

$$
g_{N}\left(\widetilde{\nabla}_{K} L, J Z\right)=g_{N}\left(v \widetilde{\nabla}_{K} L+T_{K} w L+\eta(w L) E, Z\right)
$$

which give proof. 
Corollary 4.9. Let $F$ be a semi-invariant Riemannian submersion with SSNMC. Therefore, the total space $\mathrm{M}$ is a locally product manifold of the leaves of $\operatorname{ker} F_{*}^{\perp}$ and $\operatorname{ker} F_{*}$ if and only if

$$
\begin{gathered}
v \widetilde{\nabla}_{X} B Y+A_{X} C Y \in \Gamma\left(D_{2}\right), \\
g_{B}\left(F_{*}\left(h \widetilde{\nabla}_{X} C Y\right), F_{*}(w Z)\right)=-g_{N}\left(A_{X} B Y, J Z\right)
\end{gathered}
$$

and

$$
\begin{aligned}
v \widetilde{\nabla}_{K} L+T_{K} w L+\eta(w L) E \in & \Gamma\left(D_{1}\right), \\
g_{B}\left(F_{*}\left(T_{K} \phi L\right), F_{*}(C X)\right)+g_{B}\left(F_{*}\left(h \widetilde{\nabla}_{K} w L\right), F_{*}(C X)\right)= & -g_{N}\left(v \widetilde{\nabla}_{K} \phi L, B X\right)-g_{N}\left(T_{K} w L, B X\right) \\
& -\eta(\phi L) g_{N}(K, B X)
\end{aligned}
$$

for all $X, Y \in \Gamma\left(\operatorname{ker} F_{*}^{\perp}\right), K, L \in \Gamma\left(\operatorname{ker} F_{*}\right)$ and $Z \in \Gamma\left(D_{2}\right)$.

\section{Conclusion}

Riemannian submersions and SSNMC have an important application for many sciences such as physics and mathematical physics. Researchers have increased studies on this field from different areas in recent years. In this paper, the idea of examining Riemann submersion with different connections is emphasized. We defined and studied Riemannian submersions with SSNMC for the first time. We investigated geometry of foliatons with SSNMC. The works on this subject will be useful tools for the applications of Riemannian submersion with different connections.

\section{Conflicts of Interest}

The author declares no conflict of interest.

\section{References}

[1] B. O'Neill, The Fundamental Equations of a Submersion, Michigan Mathematics Journal 13 (1966) 458-469.

[2] A. Gray, Pseudo-Riemannian Almost Product Manifolds and Submersions, Journal of Mathematics and Mechanics 16 (1967) 715-737.

[3] M. A. Akyol, G. Ayar, New Curvature Tensors Along Riemannian Submersion, Arxiv:2007.07814v1.

[4] M. A. Akyol, Conformal Semi-Slant Submersions, International Journal of Geometric Methods in Modern Physics 14(7) (2017) 1750114.

[5] M. A. Akyol, R. Sarı, Semi Slant $\xi^{\perp}-$ Riemannian Submersion, Mediterranean Journal of Mathematics 14 (2017) Article No. 234.

[6] K. S. Park, R. Prasad, Semi-Slant Submersions, Bulletin Korean Mathematical Society 50(3) (2013) 951-962.

[7] M. A. Akyol, Y. Gündüzalp, Hemi-Slant Submersions from Almost Product Riemannian Manifolds, Gulf Journal of Mathematics 4(3) (2016) 15-27.

[8] R. Sarı M. Akyol, Hemi-Slant $\xi^{\perp}-$ Riemannian Submersions in Contact Geometry, Filomat $34(11)(2020) 3747-3758$.

[9] H. M. Taştan, B. Şahin, Ş. Yanan, Hemi-Slant Submersions, Mediterranean Journal of Mathematics 13(4) (2016) 2171-2184. 
[10] M. A. Akyol, R. Sarı, E. Aksoy, Semi-Invariant $\xi^{\perp}$-Riemannian Submersions from Almost Contact Metric Manifolds, International Journal of Geometric Methods in Modern Physics 14 (2017) 1750074 .

[11] K. S. Park, H-Semi-Invariant Submersions, Taiwanese Journal of Mathematics 16 (2012) 5 18651878.

[12] B. Şahin, Semi-Invariant Riemannian Submersions from Almost Hermitian Manifolds, Canadian Mathematical Bulletin 56 (2011) 173-183.

[13] S. Ali, T. Fatima, Anti-Invariant Riemannian Submersions from Nearly Kaehler Manifolds, Filomat 27(7) (2013) 1219-1235.

[14] J. W. Lee, Anti-invariant $\xi^{\perp}$-Riemannian Submersions from Almost Contact Manifolds, Hacettepe Journal of Mathematics and Statistics 42(3) (2013) 231-241.

[15] B. Şahin, Anti-invariant Riemannian submersions from almost Hermitian manifolds, Central European Journal of Mathematics 3 (2010) 437-447.

[16] A. Friedmann, J. A. Schouten, Uber die Geometrie der Halbsymmetrischen Ubertragungen, Math Zeitschrift, 21 (1924) 211-233.

[17] H. A. Hayden, Subspaces of a Space with Torsion, Proceedings of the London Mathematical Society 34 (1932) 27-50.

[18] K. Yano, On Semi-Symmetric Metric Connection, Revue Roumaine de Mathematiques Pures et Appliquees 15 (1970) 1579-1586.

[19] N. S. Agashe, M. R. Chafle, A Semi-Symmetric Non-Metric Connection on a Riemannian Manifold, Indian Journal of Pure and Applied Mathematics 23(6) (1992) 399-409.

[20] G. Ayar, D. Demirhan, Ricci Solition on Nearly Kenmotsu Manifolds with Semi-Symmetric Metric Connection, Journal of Engineering Technology and Applied Sciences 4(3) (2019) 131-140.

[21] M. Altın, Projective Curvature N(k) Contact Metric Manifold Admitting Semi-Symetric NonMetric Connection, Fundamental Journal of Mathematics and Applications 3(2) (2020) 94-100.

[22] M. A. Akyol, R. Sarı, On CR-Submanifolds of a S-Manifold Endowed with a Semi-Symmetric non Metric Connection, Communications Faculty of Sciences University of Ankara Series A1 Mathematics and Statistics 65(1) (2016) 171-185.

[23] R. Sarı, M. Akyol, E. Aksoy Sarı, Some Properties of CR-Submanifolds of an S-Manifold with a Semi-Symmetric Metric Connection, Celal Bayar University Journal of Science 13(3) (2017) $729-736$.

[24] A. T. Vanlı İ. Ünal, D. Özdemir, Normal Complex Contact Metric Manifolds Admitting a Semi Symmetric Metric Connection, Applied Mathematics and Nonlinear Sciences 5(2) (2020) 49-66.

[25] A. T. Vanlı R. Sarı On Semi-Invariant Submanifold of a Generalized Kenmotsus Manifold Addmiting a Semi-Symmetric Non -Metric Connection, Pure and Applied Mathematics Journal $4(1-2)(2015)$ 14-18.

[26] İ. Ünal, On Submanifolds of N(k)-Quasi Einstein Manifolds with Semi-Symmetric Metric Connection, Universal Journal of Mathematics and Applications 3(4) (2020) 167-172.

[27] M. A. Akyol, S. Beyendi, Riemannian Submersions Endowed with a Semi-Symmetric Non-Metric Connection, Konuralp Journal of Mathematics, 6(1) (2018) 188-193.

[28] P. Baird, J. C. Wood., Harmonic Morphisms between Riemannian Manifolds, London Mathematical Society Monographs, 29, Oxford University Press, the Clarendon Press. Oxford, 2003. 